\title{
Estimation of Hydrodynamic Derivatives of Full-Scale Submarine using RANS Solver
}

\author{
Tien Thua Nguyen", Hyeon Kyu Yoon ${ }^{*}$, Youngbum Park ${ }^{* *}$ and Chanju Park \\ "Eco-friendly Offshore Plant FEED, Changwon National University, Changwon, Korea \\ ${ }^{*}$ Agency for Defense Development, Daejeon, Korea
}

KEY WORDS: Maneuverability, Virtual PMM tests, Hydrodynamic derivatives, Full-scale, RANS solver

\begin{abstract}
It is necessary to predict hydrodynamic derivatives when assessing the maneuverability of a submarine. The force and moment acting on the vehicle may affect its motion in various modes. Conventionally, the derivatives are determined by performing captive model tests in a towing tank or applying a system identification method to the free running model test. However, a computational fluid dynamics (CFD) method has also become a possible tool to predict the hydrodynamics. In this study, virtual captive model tests for a full-scale submarine were conducted by utilizing a Reynolds-averaged Navier-Stokes solver in ANSYS FLUENT version 18.2. The simulations were carried out at design speed for various modes of motion such as straight forward, drift, angle of attack, deflection of the rudder, circular, and combined motion. The hydrodynamic force and moment acting on the submarine appended rudders and stern stabilizers were then obtained. Finally, hydrodynamic derivatives were determined, and these could be used for evaluating the maneuvering characteristics of the submarine in a further study.
\end{abstract}

\section{Introduction}

Maneuverability is one of the most important performances of a marine vehicle which plays a vital key in the safety of navigation and efficient service. Unlike the floating vehicle, the submarine must be controlled in the six degrees of freedom. Hence, the configuration of control surfaces is also more complex to meet the demand of its controllability. Therefore, assessment of maneuverability of the submarine with control surfaces is required as well. In order to evaluate the maneuvering characteristics at the design stage, it is necessary to predict hydrodynamic force and moment acting on the vehicle in various modes of motion. It can be categorized that there are available four methods for estimating the hydrodynamics. Semi-empirical methods have been used for calculating the hydrodynamic derivatives, but these have just simple form and deal with only main design particulars of the submarine(Mackay, 2007). In addition, the panel method was developed to predict the hydrodynamic characteristics of the underwater vehicle. Nevertheless, this method did not take into account the effect of viscosity, so it could deal with only added mass and added moment of inertia(Iskender et al., 1997). Captive model tests are considered as the most accurate method to determine all linear and nonlinear hydrodynamic derivatives used for mathematical modeling of hydrodynamic force and moment(Park et al., 2017; Han et al., 2017). However, it also has some disadvantages such as the limitation of facilities, the high cost of performance, and the scale effects.

On the other hand, Computational fluid dynamics(CFD) methods are becoming the alternative solution for compromising the disadvantages of the other methods. It not only includes the viscous effects but also can model the captive model tests virtually with good accuracy and economy as well. There are three main approaches to simulate turbulent flow over the submarine, which are Direct numerical simulation(DNS), Large eddy simulation(LES), and Reynolds-averaged Navier-Stokes(RANS). DNS is the most accurate of approaches as it numerically solved Navier-Stokes equations without any turbulence model, but it requires enormous computational resources. The LES or hybrid LES-RANS approach are computed directly and only small scale motions are modeled. It is more accurate than the RANS approach since the large eddies are captured in full detail directly. However, these approaches are many times more computational expense than RANS approach. The RANS is the most common approach to turbulence modeling using the Reynolds averaging procedure. Nonetheless, it gives bad results when there is swirl or streamline curvature.

Received 1 August 2018, revised 24 September 2018, accepted 18 October 2018

Corresponding author Hyeon Kyu Yoon: +82-55-213-3683, hkyoon@changwon.ac.kr ORCID: http://orcid.org/0000-0001-6639-0927 It is noted that this paper is revised edition based on proceedings of KMRTS 2018 in KAIST.

(c) 2018, The Korean Society of Ocean Engineers

This is an open access article distributed under the terms of the creative commons attribution non-commercial license (http://creativecommons.org/licenses/by-nc/3.0) which permits unrestricted non-commercial use, distribution, and reproduction in any medium, provided the original work is properly cited. 
Regarding the use of RANS calculation in the design of submarine, Toxopeus(2008) and Zeng and Zhu(2010) demonstrated that RANS-based simulation could be used to predict the hydrodynamics of the scaled DARPA SUBOFF submarine in forward and oblique motions at small drift angle. In addition, Pan et al.(2012) proposed an approach for simulating the small drift motion and the harmonic motion of the same submarine with full appendage using the unsteady RANS-based simulation and the dynamic mesh technique. To solve the rotational motion by CFD method, Wu et al.(2015) employed the hybrid reference frames method to model the circular motion of the DARPA SUBOFF submarine. These researches show that the results of the RANS-based simulation are in good agreement with the experimental data. However, the simulations were mainly carried out for the scaled model with a few motion modes.

In the present paper, the RANS-based simulation for a full-scale submarine with appendages is done at straight motion, oblique motion, and rotational motion for both single mode and combined mode in the horizontal plane and vertical plane. The obtained force and moment of the simulations are then analyzed and non-dimensional hydrodynamic derivatives are estimated using least square method for maneuvering assessment in the further study.

\section{Case study}

\subsection{Objective}

The submarine shape in this paper is modeled following the outline of the submarine type with $85.0 \mathrm{~m}$ long, $7.7 \mathrm{~m}$ wide, and $6.7 \mathrm{~m}$ depth. Conning tower and rudder are created from NACA0020 profile, and the NACA0018 profile is used for the remained control surfaces. Fig.
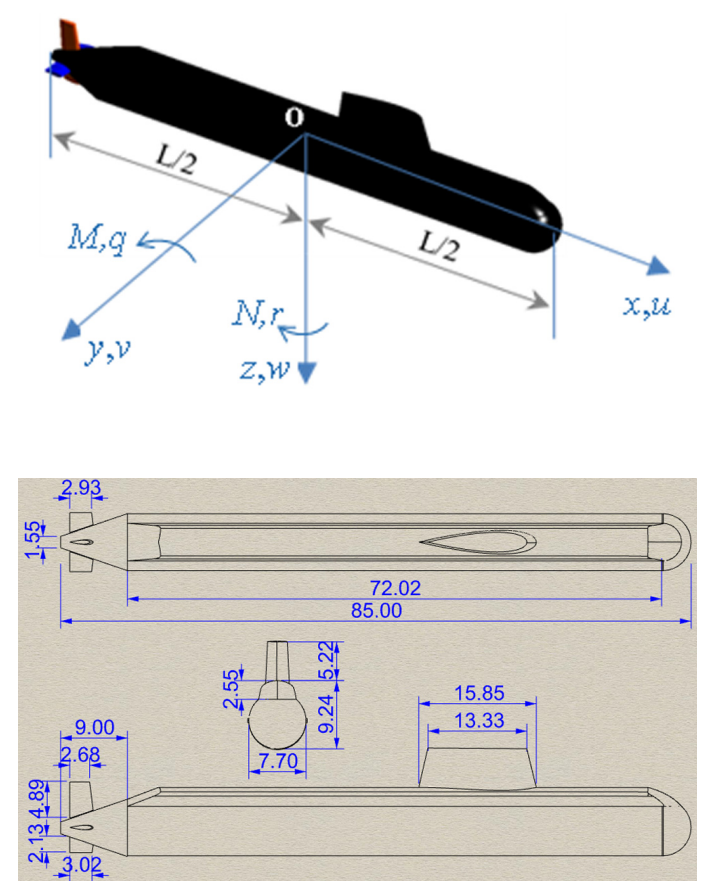

Fig. 1 Geometry and coordinate system
1 shows the definition of body-fixed coordinate system and outline drawing of the submarine which is used in this study.

\subsection{Test conditions}

In order to estimate the hydrodynamic derivatives, the virtual captive model tests of the vehicle are implemented for a series of motion modes at Reynolds number of $8.7099602 \mathrm{E}+08$. The definition of the submarine motions is described in Fig. 2, where $\alpha, \beta, \delta$ and $\omega$ are called the angle of attack(AOA), drift angle, and deflection of control surfaces, and rotational velocity, respectively. Fig. 2(a) shows the definitions of straight running $(\alpha, \beta, \delta=0)$, oblique $(\alpha$ or $\beta \neq 0, \delta=0$ ), and the deflection of control surfaces $(\alpha, \beta$ $=0, \quad \delta \neq 0$ ). The circular motion $(\alpha, \beta=0)$ and combined circular motion $(\alpha, \beta \neq 0)$ are illustrated in Fig. 2(b). Following the International towing tank conference(ITTC) recommendations on Captive Model Tests Procedure(ITTC, 2014), the computing conditions of these cases are selected as shown in Table 1.

The velocities and hydrodynamic force and moment obtained from the simulations are presented in the dimensionless form as follows,

$$
\begin{aligned}
& u^{\prime}\left(v^{\prime}, w^{\prime}\right)=\frac{u(v, w)}{V} q^{\prime}\left(r^{\prime}\right)=\frac{q(r) \times L}{V} \\
& X^{\prime}\left(Y^{\prime}, Z^{\prime}\right)=\frac{X(Y, Z)}{\frac{1}{2} \rho L^{2} U^{2}} M(N)=\frac{M(N)}{\frac{1}{2} \rho L^{3} U^{2}}
\end{aligned}
$$

where $u, v$ and $w$ are the velocity components along $x, y$ and $z$-axes, respectively. $q$ and $r$ are the angular velocities of $y$ and $z$ axes. $X$, $Y$ and $Z$ are the hydrodynamic forces along $x, y$ and $z$-axes, respectively. $M$ and $N$ are hydrodynamic moments about $y$ and $z$-axes.

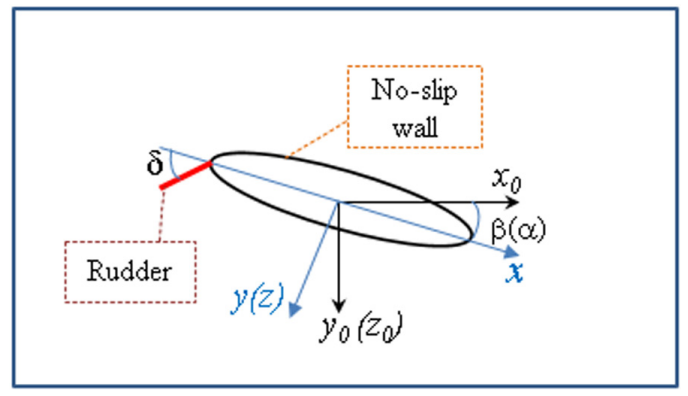

(a) Obquile motion

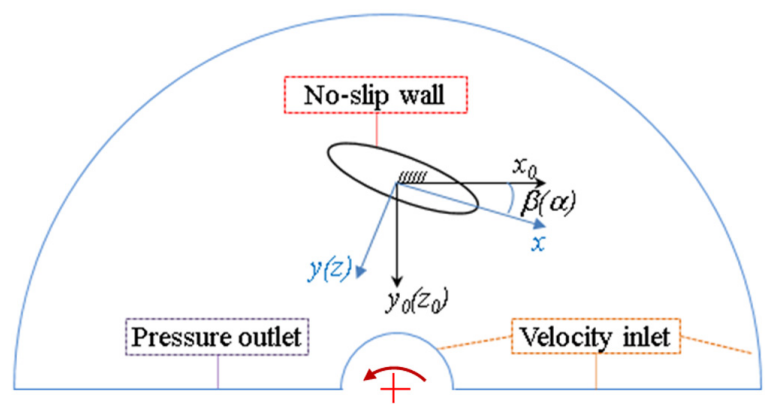

(b) Rotational motion

Fig. 2 Definition of motions 
Table 1 Test conditions

\begin{tabular}{|c|c|c|c|c|}
\hline Case & $\begin{array}{c}\alpha \\
{[\mathrm{deg} .]}\end{array}$ & $\begin{array}{c}\beta \\
{[\mathrm{deg} .]}\end{array}$ & $\begin{array}{c}\delta \\
{[\mathrm{deg} .]}\end{array}$ & $\begin{array}{l}\omega \\
{[-]}\end{array}$ \\
\hline Static drift & 0 & $-2,-4,-6,-8,-12$ & 0 & 0 \\
\hline Static angle of attack & $0, \pm 2, \pm 4, \pm 6, \pm 8$ & 0 & 0 & 0 \\
\hline Static rudder $\left(\delta=\delta_{\mathrm{r}}\right)$ & 0 & 0 & $0 \div 35$, interval 5 & 0 \\
\hline Stern stabilizer $\left(\delta=\delta_{\mathrm{s}}\right)$ & 0 & 0 & $-25 \div 25$, interval 5 & 0 \\
\hline $\begin{array}{l}\text { Horizontal circular motion(HCM) } \\
\left(\omega^{\prime}=r^{\prime}\right)\end{array}$ & 0 & 0 & 0 & $\begin{array}{l}0.12,0.17,0.21 \\
\quad 0.25,0.29\end{array}$ \\
\hline $\begin{array}{l}\text { Vertical circular motion(VCM) } \\
\qquad\left(\omega^{\prime}=q^{\prime}\right)\end{array}$ & 0 & 0 & 0 & $\begin{array}{l} \pm 0.12, \pm 0.17, \pm 0.21 \\
\quad \pm 0.25, \pm 0.29\end{array}$ \\
\hline Combined drift-HCM & 0 & $\pm 3, \pm 5, \pm 7, \pm 9$ & 0 & $0.21,0.25,0.29$ \\
\hline Combined AOA-VCM & $-3,-5,-7,-9$ & 0 & 0 & $0.21,0.25,0.29$ \\
\hline
\end{tabular}

\subsection{Numerical modeling}

The rectangular domain and cylindrical domain covering the submarine are generated for simulating the straight and oblique motion, and the circular motion, respectively. Its dimensions are chosen to be able to eliminate the effects of wall boundaries as well as reverse flow at the inlet and outlet flow conditions. Dimensions of the rectangular domain are $5 \mathrm{~L}$ in length, $4 \mathrm{~L}$ in width, and $3 \mathrm{~L}$ in height. These are $0.2 \mathrm{~L}, 5 \mathrm{~L}$, and $3 \mathrm{~L}$ for inner radius, outer radius, and thickness of the cylindrical domain. In addition, physical conditions are applied for the boundary of the domain. The front face and back face are correspondingly assigned to velocity inlet and pressure outlet. Slip condition is set for the side walls and the no-slip condition is specified for the appendages and hull surfaces. The top face and bottom face of the domain is considered as slip wall conditions.

The fluid domain is then discretized in a number of tetrahedral elements for the numerical process. Prism layer is used for modeling boundary layer of fluid flow surrounding the hull. The height of the first element next to the body's wall is created to satisfy $y^{+}$value of the boundary layer. The value of $y^{+}$can be set about 3,000-5,000 for $\mathrm{Re}=1.0 \mathrm{E}+9(\mathrm{Oh}$ and Kang, 1992), and the boundary thickness of submarine in the trial(William, 1974) is in fair agreement with the empirical relation of Granville. Using these two references, the estimated first grid height is $9.0 \mathrm{~mm}$ corresponding to $y^{+}$of 2,500.

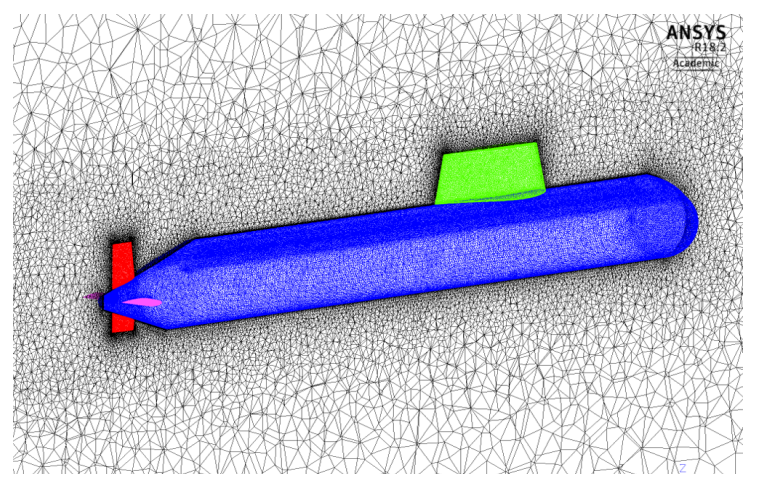

(a) Obquile motion
Fig. 3 shows the mesh of submarine in the fluid domain.

The ANSYS FLUENT software is applied for simulating the single motion and the coupled motion of the submarine in steady state. An incompressible RANS solver is opted for modeling fluid flow in these cases. According to ITTC Practical Guidelines for Ship CFD Applications(ITTC, 2011), the two-equation models have shown to be able to give an accurate prediction in ship hydrodynamics. Hence, the realizable k-epsilon turbulence model is chosen for modeling fluid flow around the submarine. Also, the second order upwind scheme is used for determining face values from interpolation of cell center values with the good accuracy and robust. Semi-implicit pressure link equations(SIMPLE) algorithm is employed to obtain pressure field and face flux by solving the momentum equation iteratively(ANSYS, 2017). Evaluation of the gradients and derivatives is done using the least square cell-based method.

The straight and oblique motions of the submarine are simulated in stationary reference frame while the steady circular motions and combined motions are carried out in moving reference frame. Following the method(ANSYS, 2017), the relative velocity vectors are defined as follows,

$$
\begin{aligned}
& \overrightarrow{v_{r}}=\vec{v}-\overrightarrow{u_{r}} \\
& \overrightarrow{u_{r}}=\overrightarrow{v_{t}}+\vec{\omega} \times \vec{R}
\end{aligned}
$$

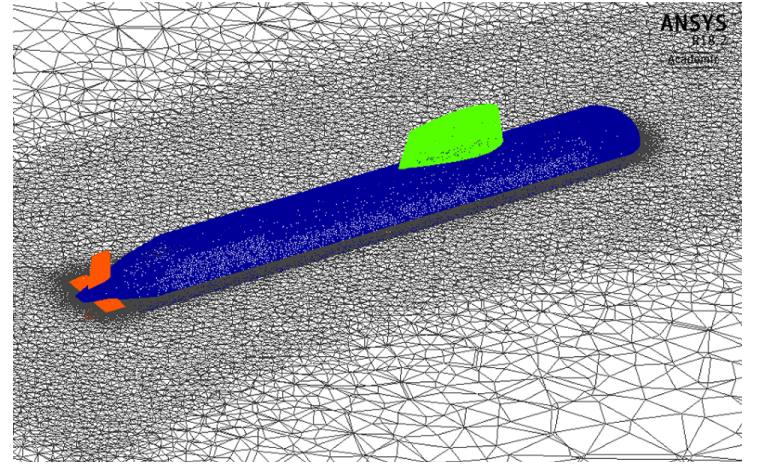

(b) Circular motion

Fig. 3 Mesh on submarine suface and center plane 
where $v_{r}$ stands for the fluid relative velocity, $v$ denotes the fluid absolute velocity, $u_{r}$ is the velocity of the moving coordinate system including translational velocity $v_{t}$ and rotational velocity $\omega . R$ is the radius of the motion.

\section{Results and discussions}

The full-scale model runs steadily with the design speed of 20 knots $(10.29 \mathrm{~m} / \mathrm{s})$. After the solution is converged at $10^{-5}$, the hydrodynamic forces and moments acting on the ship will be obtained. It is then nondimensionalized and fitted with polynomial functions for determining the dimensionless derivatives. The velocity-dependent derivatives can be taken from straight and oblique motions while rotary derivatives are extracted from the circular motion. The cross-coupled derivatives are attained in the combined motion simulation. These derivatives are used to establish the mathematical model of the hydrodynamic force and moment acting on the hull $(H D)$ and control surfaces $(C)$. According to Feldman(1979), the hydrodynamic forces and moments are mathematically modeled as follows:

Axial force:

$$
\begin{aligned}
X_{H D+C}= & \frac{\rho}{2} L^{4}\left[X_{q q}^{\prime} q^{2}+X_{r r}^{\prime} r^{2}+X_{r p}^{\prime} r p\right] \\
& +\frac{\rho}{2} L^{3}\left[X_{u}^{\prime} u+X_{v r}^{\prime} v r+X_{w q}^{\prime} w q\right] \ldots \\
& +\frac{\rho}{2} L^{2}\left[X_{v v}^{\prime} v^{2}+X_{w w}^{\prime} w^{2}\right] \\
& +\frac{\rho}{2} L^{2} u^{2}\left[X_{\delta r \delta \delta}^{\prime} u^{2} \delta_{r}^{2}+X_{\delta \delta \delta s}^{\prime} u^{2} \delta_{s}^{2}+X_{\delta b \delta b}^{\prime} u^{2} \delta_{b}^{2}\right]
\end{aligned}
$$

Lateral force:

$$
\begin{aligned}
Y_{H D+C}= & \frac{\rho}{2} L^{4}\left[Y_{r}^{\prime} r+Y_{p}^{\prime} p+Y_{|p| p}^{\prime} p|p|+Y_{p q}^{\prime} p q+Y_{q r}^{\prime} q r\right] \\
& +\frac{\rho}{2} L^{3}\left[Y_{v}^{\prime} \dot{v}+Y_{v q}^{\prime} v q+Y_{w p}^{\prime} w p\right] \ldots \\
& +\frac{\rho}{2} L^{3}\left[Y_{r}^{\prime} u r+Y_{p}^{\prime} u p+Y_{|r| \delta r}^{\prime} u|r| \delta_{r}\right] \\
& +\frac{\rho}{2} L^{2}\left[Y_{*}^{\prime} u^{2}+Y_{v}^{\prime} u v+Y_{\delta r}^{\prime} u^{2} \delta_{r}\right]
\end{aligned}
$$

Normal force :

$$
\begin{aligned}
Z_{H D+C}= & \frac{\rho}{2} L^{4} Z_{q}^{\prime} \dot{q}+\frac{\rho}{2} L^{3}\left[Z_{w}^{\prime}{ }_{w} w+Z_{v p}^{\prime} v p+Z_{q}^{\prime} u q\right] \ldots \\
& +\frac{\rho}{2} L^{2}\left[Z_{*}^{\prime} u^{2}+Z_{w}^{\prime} u w+Z_{w w}^{\prime}\left|w\left(v^{2}+w^{2}\right)^{2}\right|\right] \\
& +\frac{\rho}{2} L^{2}\left[Z_{\delta s}^{\prime} u^{2} \delta_{s}+Z_{\delta b}^{\prime} u^{2} \delta_{b}\right]
\end{aligned}
$$

Roll moment:

$$
\begin{aligned}
K_{H D+C}= & \frac{\rho}{2} L^{5}\left[K_{p}^{\prime} p+K_{r}^{\prime} r+K_{q r}^{\prime} q r+K_{p|p|}^{\prime} p|p|\right] \\
& +\frac{\rho}{2} L^{4}\left[K_{p}^{\prime} u p+K_{r}^{\prime} u r+K_{v}^{\prime} v+K_{w p}^{\prime} w p\right] \ldots \\
& +\frac{\rho}{2} L^{3}\left[K_{*}^{\prime} u^{2}+K_{v R}^{\prime} u v\right]+\frac{\rho}{2} L^{3} K_{\delta r}^{\prime} u^{2} \delta_{r}
\end{aligned}
$$

Pitch moment:

$$
\begin{aligned}
M_{H D+C}= & \frac{\rho}{2} L^{5}\left[M_{\dot{q}}^{\prime} \dot{q}+M_{r p}^{\prime} r p\right]+\frac{\rho}{2} L^{4}\left[M_{\dot{w}}^{\prime} \dot{w}+M_{q}^{\prime} u q\right] \\
& +\frac{\rho}{2} L^{3}\left[M_{*}^{\prime} u^{2}+M_{w}^{\prime} u w+M_{w|w| R}^{\prime} w\left|\left(v^{2}+w^{2}\right)^{\frac{1}{2}}\right|\right] \ldots \\
& +\frac{\rho}{2} L^{3}\left[M_{\delta s}^{\prime} u^{2} \delta_{\mathrm{s}}+M_{\delta b}^{\prime} u^{2} \delta_{\mathrm{b}}\right]
\end{aligned}
$$

Yaw moment:

$$
\begin{aligned}
N_{H D+C}= & \frac{\rho}{2} L^{5}\left[N_{\dot{r}}^{\prime} \dot{r}+N_{\dot{p}}^{\prime} \dot{p}+N_{p q}^{\prime} p q\right] \\
& +\frac{\rho}{2} L^{4}\left[N_{\dot{v}}^{\prime} \dot{v}+N_{r}^{\prime} u r+N_{|r| \delta r}^{\prime} u|r| \delta_{r}+N_{p}^{\prime} u p\right] \ldots \\
& +\frac{\rho}{2} L^{3}\left[N_{*}^{\prime} u^{2}+N_{v}^{\prime} u v+N_{v|v| R}^{\prime} v\left|\left(v^{2}+w^{2}\right)^{\frac{1}{2}}\right|\right] \\
& +\frac{\rho}{2} L^{3}\left[N_{\delta r}^{\prime} u^{2} \delta_{r}+N_{\delta r \eta}^{\prime} u^{2} \delta_{r}\left(\eta-\frac{1}{C}\right) C\right]
\end{aligned}
$$

where eg. $X_{\dot{u}}^{\prime}=\frac{\partial X^{\prime}}{\partial \dot{u}}, \quad K_{p}^{\prime}=\frac{\partial K^{\prime}}{\partial \dot{p}}, \quad X_{q q}^{\prime}=\frac{\partial^{2} X^{\prime}}{\partial q^{2}}, \quad K_{p}^{\prime}=\frac{\partial^{2} K^{\prime}}{\partial p}, \quad X_{v r}^{\prime}=\frac{\partial^{2} X^{\prime}}{\partial v \partial \partial r}$, $K_{w p}^{\prime}=\frac{\partial^{2} K^{\prime}}{\partial w \partial p}, X_{\delta_{r} \delta_{r}}^{\prime}=\frac{\partial^{2} X^{\prime}}{\partial \delta_{r}^{2}}, K_{\delta_{r}}^{\prime}=\frac{\partial^{2} K^{\prime}}{\partial \delta_{r}}$ etc, are dimensionless added masses and added masses moments of inertia, velocity-dependent derivatives and rotary-dependent derivatives, coupled derivatives, derivatives of control surfaces, respectively.
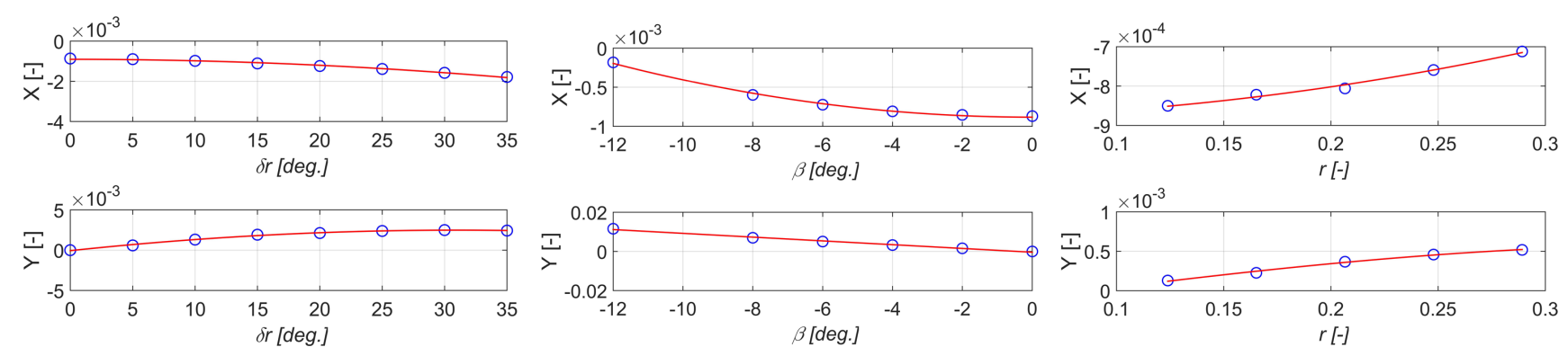

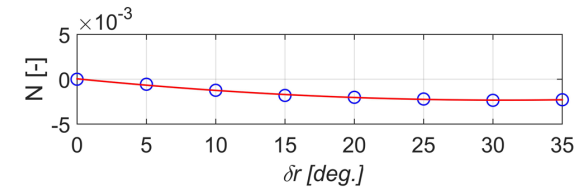

(a) Static rudder case

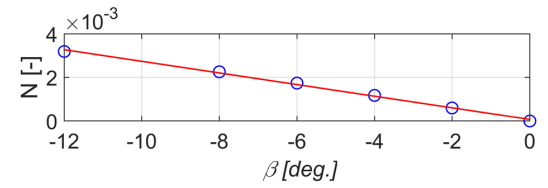

(b) Static drift case

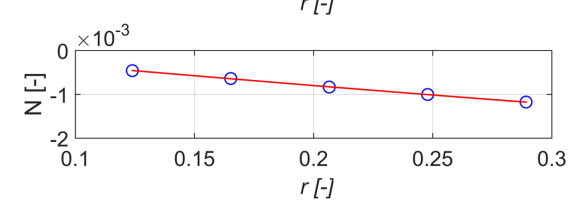

(c) Circular motion in xy plane

Fig. 4 Hydrodynamic force against motion variables in horizontal plane 

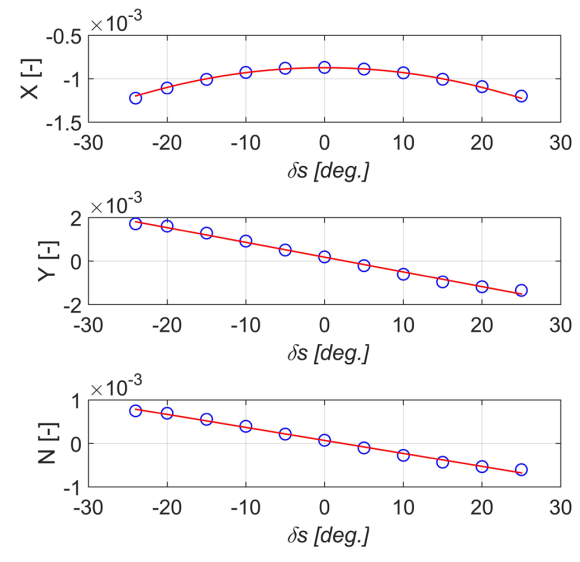

(a) Stern plane case
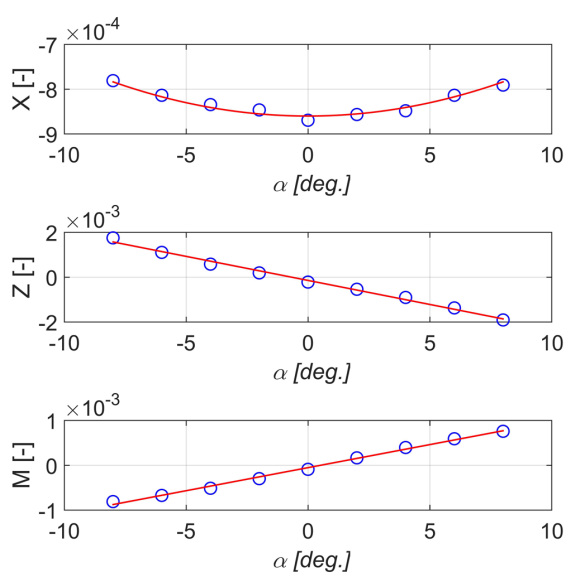

(b) Angle of attack case
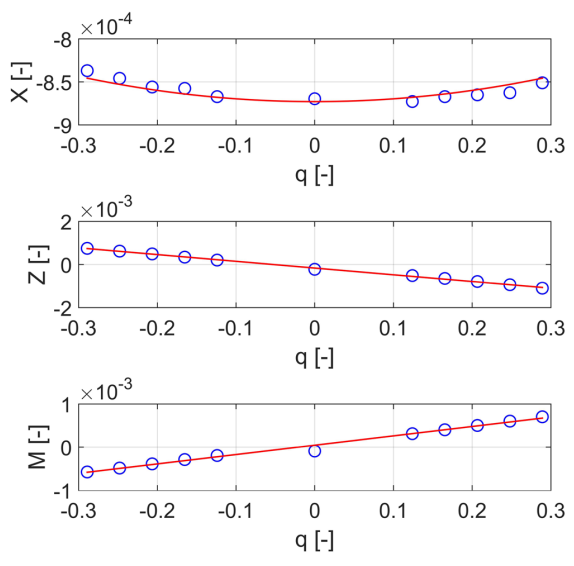

(c) Circular motion in $\mathrm{xz}$ plane

Fig. 5 Hydrodynamic force against motion variables in vertical plane

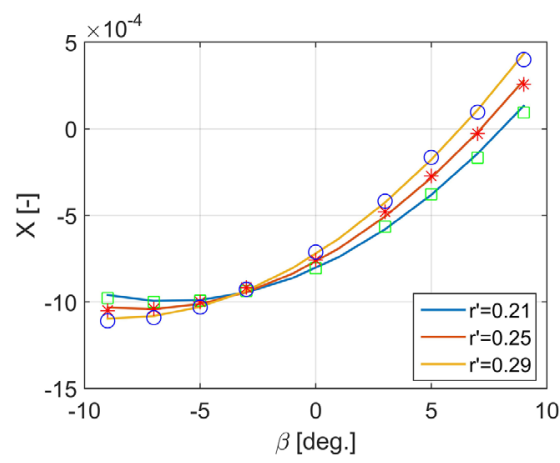

(a) Surge force

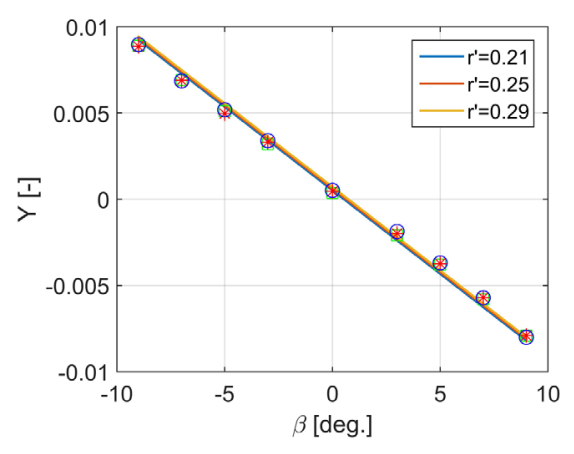

(b) Sway force

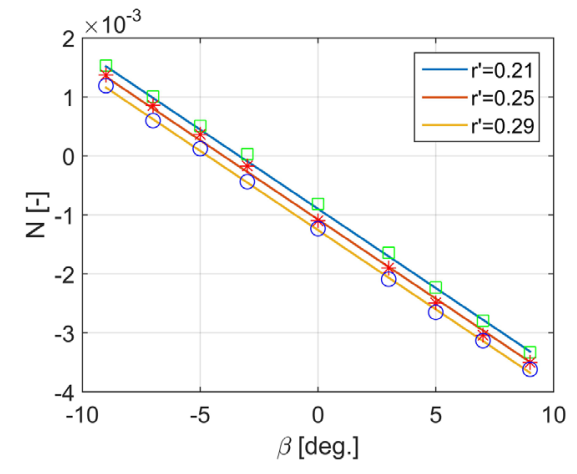

(c) Yaw moment

Fig. 6 Force and moment acting on the submarine in combined drift-circular motion

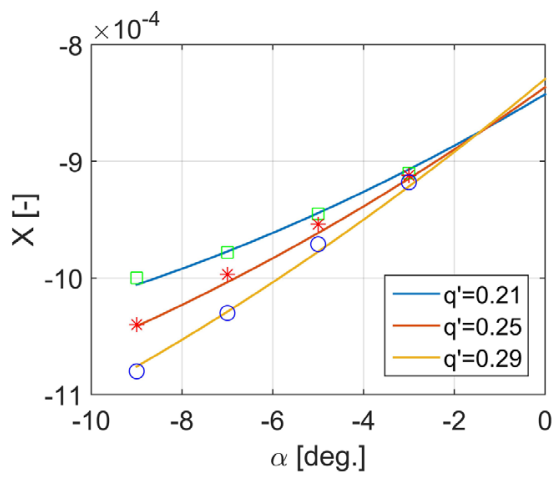

(a) Surge force

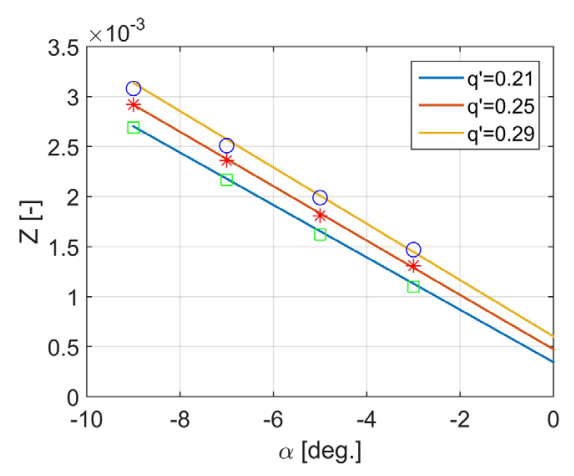

(b) Normal force

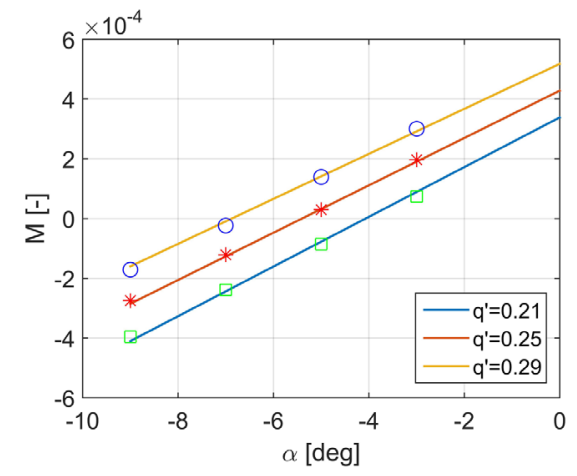

(c) Pitch moment

Fig. 7 Force and moment acting on the submarine in combined AOA-circular motion

The ship is symmetrical about the vertical center plane, the calculations are implemented in the positive range of drift angles and rudder angles. Fig. 4 shows the forces and moments acting on the ship and rudder in cases of the static rudder, drift motion, and circular motion(CM) in the horizontal plane. The hydrodynamic force acting on the ship in cases of stern stabilizer, angle of attack, and circular motion in the vertical plane is shown in the Fig. 5. It can be seen that the forces acting on the submarine in case of positive attack angle are greater than the one for the negative side. The discrepancy between the two case decreases with increasing of the attack angle and it might be caused by the asymmetry in the vertical plane of the body as well as the effect of the lifting surface at the stern.

The coupled derivatives are determined from the combined motion by subtracting forces and moments acting on the ship in the single motions from the forces and moments acting on the ship in the combined motion. The residual forces and moments are approximated using the least square method. However, the results of the combined drift-circular motion show that linear function of sway velocity and 
yaw rate is enough to represent the variation of sway force and yaw moment. On the other hand, the hydrodynamic forces of the combined angle of attack and circular motion result in two coupled derivatives. Figs. 6-7 describe the force and moment acting on the submarine and fitting curves in these two cases. The hydrodynamic derivatives obtained from the simulation in this study are listed in Table 2.

Flow pattern around the sumarine is extracted to visualize flow characteristics during its operation. Fig. 8 illustrates contours of velocity magnitude in four cross sections located at fore and aft of the submarine. It can be seen that there is a development of vortex flow behind the conning tower. It becomes stronger as the vorticity sheds from the

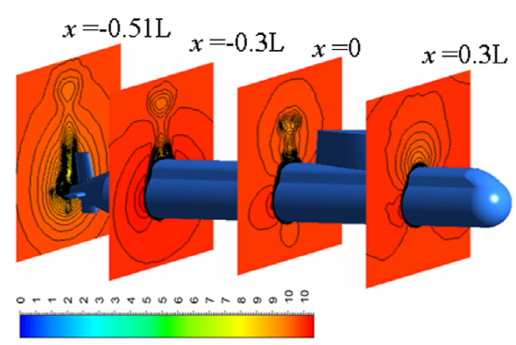

(a) Static AOA

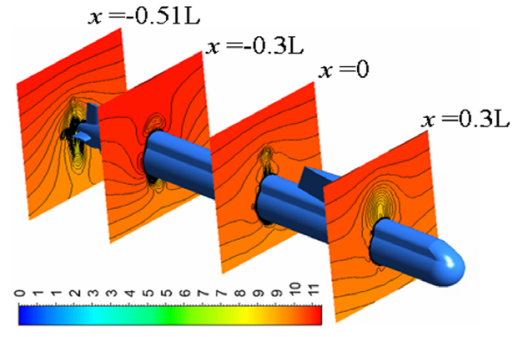

(c) Combined AOA-CMT

Fig. 8 Velocity contours in various motions

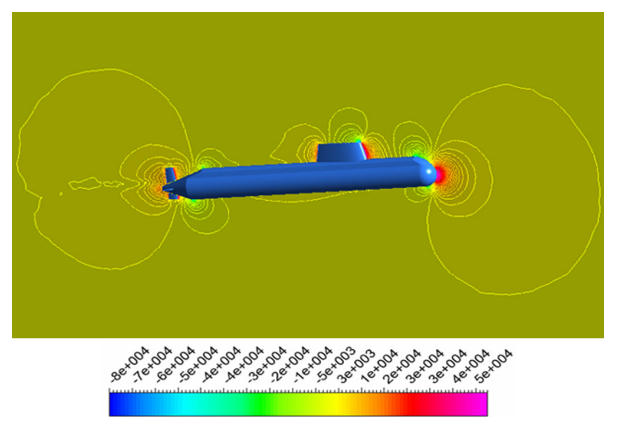

(a) Static AOA

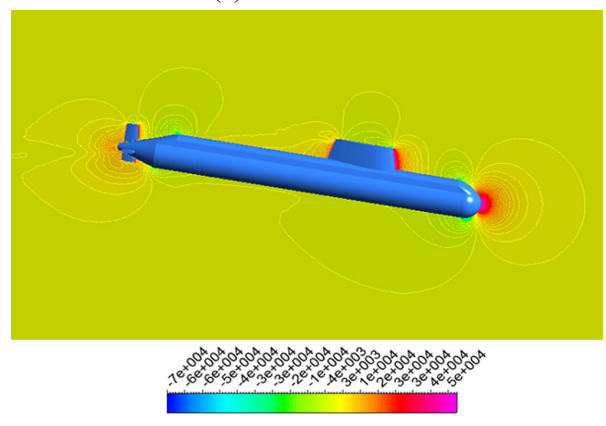

(c) Combined AOA-CMT

Fig. 9 Pressure contours in single motion and coupled motion
Table 2 Hydrodynamic derivatives $\left(\times 10^{5}\right)$

\begin{tabular}{|c|c|c|c|c|c|}
\hline Coeff. & Value & Coeff. & Value & Coeff. & Value \\
\hline$X_{q q}^{\prime}$ & 32.05 & $Y_{r}^{\prime}$ & 244.64 & $M_{q}^{\prime}$ & -217.13 \\
\hline$X^{\prime}{ }_{r r}$ & 199.89 & $Y_{v}^{\prime}$ & -5548.54 & $M^{\prime}{ }_{w}$ & 589.32 \\
\hline$X^{\prime}{ }_{v v}$ & 1591.2 & $Y^{\prime}{ }_{\delta r}$ & 734.99 & $M_{|w| q}^{\prime}$ & -545.34 \\
\hline$X^{\prime}{ }_{w w}^{\prime}$ & 182.43 & $Y^{\prime}{ }_{\delta r \mid \delta r}$ & -871.80 & $M^{\prime}{ }_{\delta s}$ & -169.69 \\
\hline$X_{w q}^{\prime}$ & -641.54 & $Z_{q}^{\prime}$ & -312.07 & $N_{r}^{\prime}$ & -436.26 \\
\hline$X^{\prime}{ }_{v r}$ & 1684.04 & $Z_{w}^{\prime}$ & -1219.8 & $N_{v}^{\prime}$ & -1538.28 \\
\hline$X^{\prime}{ }^{\prime}{ }^{\prime} \delta r$ & -243.42 & $Z^{\prime}{ }_{w|q|}$ & -1358.48 & $N^{\prime}{ }_{\delta r}$ & -687.60 \\
\hline$X^{\prime}$ os $\delta s$ & -179.82 & $Z^{\prime}{ }_{\delta s}$ & -385.40 & $N^{\prime}{ }_{\delta r \mid} \delta r \mid$ & 819.90 \\
\hline
\end{tabular}

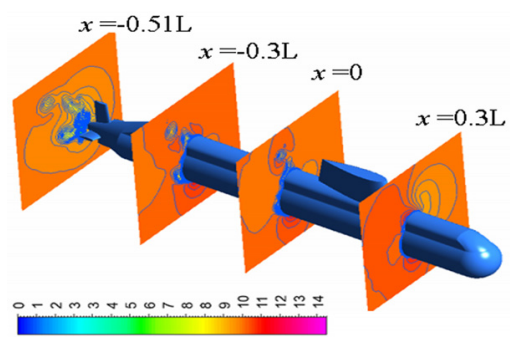

(b) Static drift

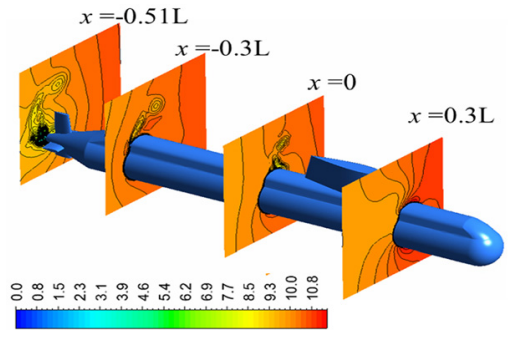

(d) Combinned drift-CMT

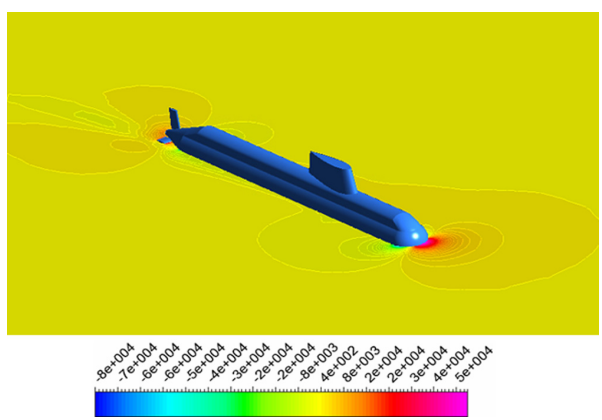

(b) Static drift

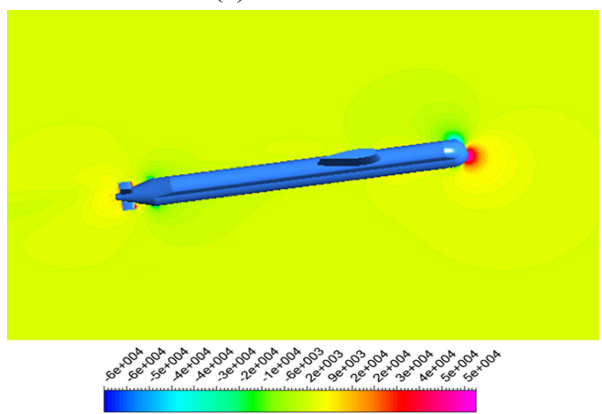

(d) Combinned drift-CMT 
conning tower to the stern. The vortex generates the asymmetric forces acting on the submarine which afftects diving motion.

Fig. 9 shows the pressure fields of the vehicle when it travels in the angle of attack, drift, the combined angle of attack and circular, and combined drift and circular motions. The slices in the center plane and horizontal plane are colored by pressure distribution of flow around the hull and appendage. It is observed that the oblique and the combined motion cause asymmetry pressure field which leads to sway force, heave force, pitch moment, and yaw moment.

\section{Conclusions}

In this study, CFD RANS-based simulation of a full-scale submarine has been implemented when it runs straightly and rotationally. The straight motion and obquile motion is simulated in the stationary reference frame and the circular motion is modeled using the moving reference frame. The simulation results are fitted to curves and surfaces using the least square method. As the results, the velocity-dependent derivatives, rotary-dependent derivatives, and cross-coupled derivatives are estimated. These derivatives may be used to confirm the submarine's maneuverability in its preliminary design. In the future, the simulation results will be verified with the experimental results.

\section{Acknowledgement}

This study was supported by the research project "Scenario and modeling of submarine's maneuvering and motion for development of navigation algorithm", of Agency for Defense Development.

\section{References}

Ansys Inc., 2017. Fluent Theory Guide 18.2.

Feldman, J., 1979. DTNSRDC Revised Standard Submarine Equations of Motion. Report, David W. Taylor Naval Ship Research and Development Center, DTNSRDC/SPD-0393-09. Han, J.H., Jeong, J.H., Lee, S.B., Jang, K.Y., Lee, S.K., 2017. Dynamic Stability Analysis of a Submarine by Changing Conning Tower Position and Control Planes. Journal of Navigation and Port
Research, 41(6), 389-394.

International Towing Tank Conference(ITTC), 2011. ITTC-Recommended Procedures and Guidelines: Practical Guidelines for Ship CFD Applications.

International Towing Tank Conference(ITTC), 2014. ITTC-Recommended Procedures and Guidelines: Captive Model Test Procedures.

Iskender, S., Jan, W.C., Kennard, P.W., 1997. Application of a Panel Method to Hydrodynamics of Underwater Vehicles. Ocean Engineering, 24(6), 501-512.

Mackay, M., 2007. Semiempirical Component Based Modeling of Submarine Hydrodynamics and Systems: the DSSP21 (build 011102) Companion. Technical Report, Defense Research and Development Canada.

Oh, K.J., Kang, S.H., 1992. Full Scale Reynolds Number Effects for the Viscous Flow around the Ship Stern. Journal of Computational Mechanics, 9(2), 85-94.

Pan, Y.C., Zhang H.X., Zhou, H.X., 2012. Numerical Prediction of Submarine Hydrodynamic Coefficients Using CFD Simulation. Journal of Hydrodynamics, 24(6), 840-847.

Park, J.Y., Kim, N., Shin, Y.K., 2017. Experimental Study on Hydrodynamic Coefficients for High-incidence-angle Maneuver of a Submarine. International Journal of Naval Architecture and Ocean Engineering, 9(1), 100-113.

Toxopeus, S., 2008. Viscous-flow Calculation for Bare Hull DARPA SUBOFF Submarine at Incidence. International Shipbuilding Progress, 55(3), 227-251.

William, G.S., 1974. Turbulent Boundary Layer and Viscous Resistance of a Submarine at High Reynolds Number. Report, Naval Ship Research and Development Center.

Wu, X., Wang, Y., Huang, C., Hu, Z., Yi, R., 2015. An Effective CFD Approach for Marine-vehicle Maneuvering Simulation Based on the Hybrid Reference Frame Method. Ocean Engineering, 109, 83-92.

Zeng, G.H., Zhu, J., 2010. Study on Key Techniques of Submarine Maneuvering Hydrodynamics Prediction using Numerical Method. Proceedings of Second International Conference on Computer Modeling and Simulation, Hainan China, 83-87. 